\title{
Analysis of Slotted Sectoral Waveguides Embedded in Cylindrically Stratified Media Using Closed-Form Green's Function Representations
}

\author{
M. Kalfa ${ }^{1,2}$ and V.B. Erturk $^{1 *}$ \\ ${ }^{1}$ Dept. of Electrical and Electronics Engineering, \\ Bilkent University, TR-06800, Bilkent, Ankara, Turkey \\ ${ }^{2}$ Radar, Electronic Warfare, and Intelligence Systems Division, \\ Aselsan Inc., TR-06370, Ankara, Turkey
}

Slotted waveguide antenna arrays with dielectric covers are widely used in both military and civil applications due to their low-profile, high power handling capacity, and the ability to conform to the host platform. Conformity is especially required for air platforms where aerodynamics and radar cross section (RCS) of the vehicle are of utmost importance. For an air platform, one or more dielectric layers (monolithic or sandwich radomes) can be used to protect the antenna from the extreme flight conditions. Although the accurate and efficient design and analysis of low-profile conformal slotted waveguide arrays is of great interest, available solution methods in the literature usually suffer in terms of efficiency and memory requirements. Among the available solution methods, one of the widely used solvers are integral equation (IE) based ones that utilize the method of moments (MoM). However, the IE solvers suffer from long matrix fill times, especially when cylindrically stratified media are considered.

In this study, a slotted sectoral waveguide antenna, covered by multiple dielectric layers is analyzed with a hybrid MoM/Green's function technique in the space domain. Only the fundamental mode of propagation $\left(\mathrm{TE}_{11}\right)$ is considered. The longitudinal slots are on the broadside wall of the waveguide and are very short in the transverse direction, so that longitudinal electric fields can be neglected. The solution domain is divided into two by using fictitious magnetic current sources on the waveguide slots, while covering the slots with a perfect electric conductor (PEC), using equivalence theorem. Note that for the purposes of this study, the waveguide wall thickness is assumed to be zero. However, it can be incorporated into the problem by adding a third region which would be a sectoral cavity. The magnetic sources on the slots are expanded by piecewise sinusoid (PWS) basis functions, and the continuity of the tangential magnetic fields across the slots is used to construct the integral equation. The integral equations are then converted into matrix form using Galerkin's procedure. To compute the elements of the mutual admittance matrix, two Green's function representations for the two solution regions are used. For the waveguide interior, the dyadic Green's function components for a sectoral waveguide corresponding to longitudinal magnetic currents are rigorously derived. For the cylindrically stratified dielectric region, closed-form Green's function (CFGF) representations for magnetic currents are developed, which are valid for all source and observation points, including the source region. The proposed analysis method can be easily extended to include: slotted substrate-integrated waveguides, slotted cavity antennas, and similar aperture type antennas embedded in cylindrically stratified media.

Numerical results in the form of slot fields and input admittance for a generic slotted sectoral waveguide are presented, and compared to the results obtained from the commercially available full-wave electromagnetic solvers. 